\title{
ON APPROXIMATION TO ANALYTIC FUNCTIONS BY RATIONAL FUNCTIONS
}

\section{H. MARGARET ELLIOTT}

Let $f(z)$ be analytic in the interior of a rectifiable Jordan curve $C$ and continuous in the corresponding closed region $\bar{C}$. The relation between continuity properties of $f(z)$ on $C$ and degree of approximarion to $f(z)$ by polynomials $\pi_{n}(z)$ in $z$ of respective degrees $n, n$ $=1,2, \cdots$, has been extensively studied. In the present paper we study the relation between continuity properties of $f(z)$ on $C$ and degree of approximation to $f(z)$ on $\bar{C}$ by rational functions $r_{n}(z)$ in $z$ of respective degrees $n$ whose poles lie exterior to $C$. We thus deal with what is frequently referred to in the theory as Problem $\alpha$.

1. Definitions. By a rational function in $z$ of degree $n$ we shall mean a function $r_{n}(z)$ of the form $r_{n}(z)=\left(\sum_{j=0}^{n} a_{j} z^{j}\right) /\left(\sum_{j=0}^{n} b_{j} z^{j}\right)$, where the denominator is not identically zero. None of the $a_{j}$ is assumed to be different from zero. The degree of a rational function will always be indicated by its subscript.

The continuity properties of a function $f(z)$ to which we approximate will be described in terms of the modulus of continuity of $f(z)$ or one of its derivatives. Let $E$ be a closed bounded set in the z-plane which consists of the mutually disjoint Jordan arcs, Jordan curves, and closed Jordan regions $E_{j}, j=1, \cdots, \mu$. Let $f(z)$ be a real or complex function that is continuous on each $E_{j}$. The modulus of continuity $\omega_{j}(\delta)$ of $f(z)$ on $E_{j}$ shall be defined as the maximum of $\left|f\left(z_{1}\right)-f\left(z_{2}\right)\right|$ for all $z_{1}$ and $z_{2}$ in $E_{j}$ such that $\left|z_{1}-z_{2}\right| \leqq \delta$. The modulus of continuity $\omega(\delta)$ of $f(z)$ on $E$ shall be defined as the maximum of $\omega_{j}(\delta), 1 \leqq j \leqq \mu$. For properties of the modulus of continuity, the reader is referred to $[1, \S 2]$.

Let $E$ with boundary $C$ be a closed limited point set in the $z$-plane whose complement $K$ is connected and regular in the sense that $K$ possesses a Green's function $G(x, y), z=x+i y$, with pole at infinity. We shall denote by $C_{R}$ the locus $G(x, y)=\log R>0$ which lies in $K$.

If $C$ is any set of closed curves, we denote the sum of their closed interiors by $\bar{C}$.

The letter $k$ will be reserved for,positive integers and zero. The letters $L$ and $M$ with or without subscripts denote positive constants which may vary from one theorem and its proof to another and may

Presented to the Society, October 27, 1951; received by the editors March 31, 1952. 
depend on the point sets involved, but which are independent of $w, z, \delta, m$, and $n$. For any function $f(z), f^{(0)} \equiv f(z)$.

2. Degree of approximation. In this section from given continuity properties of a function we shall derive the existence and degree of convergence of a sequence of approximating rational functions having specified poles.

When we refer to a function $f(z)$ as being analytic in several mutually exterior regions, we do not imply that there is any relationship between the values of $f(z)$ for $z$ in different regions. Here and throughout we write "there exist polynomials (rational functions)" rather than "for each $n, n=1,2, \cdots$, there exists a polynomial (a rational function) of degree $n . "$

TheOREM 2.1. Let $C$ consist of a finite number of mutually exterior analytic Jordan curves. Let $f(z)$ be analytic in the interior of $C$ and continuous on $\bar{C}$. Suppose $f^{(k)}(z)$ exists and is continuous on $C$ with modulus of continuity $\omega(\delta), \omega(\delta) \not \equiv 0$. Let points $\alpha_{n j}, j=1, \cdots, n$; $n=1,2, \cdots$, be given on or exterior to $C_{A}, A>1$. Then there exist rational functions $r_{n}(z)$,

$$
r_{n}(z)=\frac{a_{n 0} z^{n}+a_{n 1} z^{n-1}+\cdots+a_{n n}}{\left(z-\alpha_{n 1}\right)\left(z-\alpha_{n 2}\right) \cdots\left(z-\alpha_{n n}\right)},
$$

such that

$$
\left|f(z)-r_{n}(z)\right| \leqq M \omega(1 / n) / n^{k}, \quad \text { zon } \bar{C} .
$$

The $\alpha_{n j}$ need not all be distinct or finite. We make the convention that if an $\alpha_{n j}$ is infinite, then the factor $\left(z-\alpha_{n j}\right)$ is to be replaced by unity. It will appear from the proof of Theorem 2.1 that the approximating rational functions $r_{n}(z)$ can be chosen so that $\sum_{i=0}^{n} a_{n i} \alpha_{n j}^{n-i} \neq 0, j=1, \cdots, n$. It is sufficient to assume the existence of $f^{(k)}(z)$ on the boundary merely in the one-dimensional sense.

Theorem 2.1 for the case that $C$ is a single analytic Jordan curve and $f^{(k)}(z)$ satisfies a Lipschitz condition has been stated by Sewell [2, Theorem 4.3]; his proof, however, contains an error. It is impossible to choose the quantities involved so that inequality (4.13) in Sewell's paper is satisfied.

We proceed to prove Theorem 2.1. It is known [1, Theorem 3.3] that there exist polynomials $\pi_{m}(z)$ in $z$ such that

$$
\left|f(z)-\pi_{m}(z)\right| \leqq M_{1} \omega(1 / m) / m^{k}, \quad z \text { in } \bar{C} \text {. }
$$

Let $\beta_{n j}, j=1, \cdots, n+1$, be $n+1$ points on $C$ such that the maximum of the absolute value of 


$$
\begin{aligned}
V_{n}\left(z_{1}, z_{2}, \cdots, z_{n+1}\right) \\
=\left[\prod_{i, j=1, i<j}^{n+1}\left(z_{i}-z_{j}\right)\right] /\left[\prod_{i, j=1}^{i=n+1, j=n}\left(z_{i}-\alpha_{n j}\right)\right], \quad n \geqq 2,
\end{aligned}
$$

for the $z_{j}, j=1, \cdots, n+1$, on $C$ is taken on for $z_{j}=\beta_{n j}$. If any $\alpha_{n j}$ is at infinity, the $n+1$ factors $\left(z_{i}-\alpha_{n j}\right)$ are to be replaced by unity. The $\beta_{n j}$ are the Fekete-Shen points.

Let $z$ lie on $C$; for $t$ exterior to $C$ it is easily verified that

$$
r_{n}(t, z)=\sum_{j=1}^{n+1} \frac{1}{t-\beta_{n j}} \frac{V_{n}\left(\beta_{n 1}, \cdots, \beta_{n, j-1}, z, \beta_{n, j+1}, \cdots, \beta_{n, n+1}\right)}{V_{n}\left(\beta_{n 1}, \cdots, \beta_{n, n+1}\right)}
$$

is a function of form (2.1) which interpolates to $1 /(t-z)$ in the points $\beta_{n j}$. Choose $R, 1<R<A$. It is a consequence of (2.3) and the choice of the $\beta_{n j}$ that $\left|r_{n}(t, z)\right| \leqq(n+1) / d$ for $z$ on $C$ and $t$ on $C_{R}, d$ denoting the minimum distance from $C$ to $C_{R}$. Furthermore [3, p. 185]

$$
\frac{1}{t-z}-r_{n}(t, z)=\frac{1}{t-z} \frac{\omega_{n}(z)}{\omega_{n}(t)}
$$

where $\omega_{n}(z)=\left(z-\beta_{n 1}\right) \cdots\left(z-\beta_{n, n+1}\right) /\left(z-\alpha_{n 1}\right) \cdots\left(z-\alpha_{n n}\right)$. Hence

$$
\left|\frac{1}{t-z} \frac{\omega_{n}(z)}{\omega_{n}(t)}\right| \leqq \frac{n+2}{d}, \quad z \text { on } C, t \text { on } C_{R} \text {. }
$$

Let $G\left(z, \alpha_{n j}\right)$ denote Green's function for the exterior of $C_{R}$ with pole at $z=\alpha_{n j}$. Then $w=\phi\left(z, \alpha_{n j}\right)=\exp \left[G\left(z, \alpha_{n j}\right)+i H\left(z, \alpha_{n j}\right)\right]$, where $H$ is conjugate to $G$ in the exterior of $C_{R}$, maps the exterior of $C_{R}$ onto $|w|>1$ so that the point $z=\alpha_{n j}$ goes into the point at infinity in the w-plane. On $C_{R},\left|\phi\left(z, \alpha_{n j}\right)\right|=1$. Hence

$$
\left|\frac{\omega_{n}(z)}{\omega_{n}(t)} \frac{\prod_{j=1}^{n} \phi\left(t, \alpha_{n j}\right)}{t-z}\right| \leqq \frac{n+2}{d}, \quad z \text { on } C, t \text { on } C_{R} \text {. }
$$

The functions $\left[\prod_{j=1}^{n} \phi\left(t, \alpha_{n j}\right)\right] /\left[(t-z) \omega_{n}(t)\right]$, considered as functions of $t$, for $t$ exterior to $C_{R}$ and $z$ on $C$, are analytic and their moduli are single-valued. Thus (2.4) is valid for $z$ on $C$ and $t$ exterior to $C_{R}$.

There exist constants $L_{j}$ such that $\left|\phi\left(t, \alpha_{n j}\right)\right| \geqq L_{j}>1$ for $t$ on $C_{A}$. Set $L=\min _{1 \leqq j \leqq n} L_{j}$. Then

$$
\left|\frac{\omega_{n}(z)}{\omega_{n}(t)} \frac{1}{t-z}\right| \leqq \frac{n+2}{d L^{n}}, \quad z \text { on } C, t \text { on } C_{A} .
$$


If $R_{n, m}(z)$ is the rational function of form (2.1) which interpolates to $\pi_{m}(z)$ at the points $\beta_{n j}, j=1, \cdots, n+1$, we have $[3$, p. 186]

$$
\pi_{m}(z)-R_{n, m}(z)=\frac{1}{2 \pi i} \int_{C_{A}} \frac{\omega_{n}(z)}{\omega_{n}(t)} \frac{\pi_{m}(t)}{t-z} d t, \quad z \text { on } C .
$$

From (2.2) it follows that the $\left|\pi_{m}(z)\right|$ are uniformly bounded for $z$ on $C$ and hence $\left[3\right.$, p. 77] for $z$ on $C_{A}$ we have $\left|\pi_{m}(z)\right| \leqq M_{2} A^{m}$. Thus from (2.5), (2.6) we obtain

$$
\left|\pi_{m}(z)-R_{n, m}(z)\right| \leqq M_{3} n A^{m} / L^{n}, \quad z \text { on } \bar{C} .
$$

Let $q$ be an integer such that $A / L^{q}=r, r<1$. Then for $j=0,1, \cdots$, $q-1 ; m=1,2, \cdots$, it follows that

$$
\left|\pi_{m}(z)-R_{q m+j, m}(z)\right| \leqq M m r^{m}, \quad z \text { on } C .
$$

We have $r^{m} \leqq M_{5} / m^{k+2}$ and $[1$, Theorem 3.1$] 1 / m^{k+2} \leqq M_{\sigma \omega}(1 / m) / m^{k+1}$. Hence with the aid of (2.2) we obtain

$$
\left|f(z)-R_{q m+j, m}(z)\right| \leqq M_{7} \omega(1 / m) / m^{k}, \quad z \text { on } \bar{C} .
$$

Since [1, Theorem 3.1] there exists a constant $M_{8}$ such that $\omega(1 / m) / m^{k}<M_{8} \omega(1 /(q m+j)) /(q m+j)^{k}$, the theorem follows if we define $r_{n}(z)=1 /\left(z-\alpha_{n 1}\right) \cdots\left(z-\alpha_{n n}\right)$ for $n=1,2, \cdots, q-1$ and $r_{q m+j}(z)=R_{q m+j, m}(z)$ for $j=0,1, \cdots, q-1 ; m=1,2, \cdots$.

In the case that $C$ is a single analytic Jordan curve, the above proof can be somewhat simplified by using a known lemma [3, p. 250].

3. Degree of approximation; inverse problem. We turn now to the converse question: given a sequence of rational functions which converge to a function in a certain manner, what can be said about the continuity properties of the function? We establish our results for a single closed Jordan region, but they clearly hold for a finite number of such regions which are mutually exterior.

Definition 3.1. A curve $C$ is said to be of Type $\mathrm{D}$ if (i) $C$ is a rectifiable Jordan curve such that for every arc of $C$ the ratio of the length of that arc to the length of its corresponding chord is less than a constant $A_{0}$; and (ii) there exists a number $\delta_{0}>0$ such that through any point $P$ of $C$ there is a circle of radius $\delta_{0}$ whose closed interior lies in $\bar{C}$.

To establish our results, we approximate by polynomials the rational functions which are assumed to converge to $f(z)$ in a certain manner; a known theorem on polynomial approximation then enables us to draw the desired conclusions.

We henceforth assume $\Omega(x)$ to be a real, non-negative function which is nonincreasing for $x$ sufficiently large and which is such that 
$\int \infty[\Omega(x) / x] d x$ exists.

The following known result on polynomial approximation [1, Theorem 5.3] will be utilized:

TheOREM 3.1. Let $f(z)$ be defined in $\bar{C}$, where $C$ is a curve of Type D; let polynomials $\pi_{n}(z)$ in $z$ exist such that

$$
\left|f(z)-\pi_{n}(z)\right| \leqq \Omega(n) / n^{h}, \quad z \text { in } \bar{C} .
$$

Then $f(z)$ is analytic in the interior of $C$ and continuous on $\bar{C} ; f^{(k)}(z)$ exists and is continuous on $\bar{C}$ with modulus of continuity $\omega(\delta)$ which satisfies the condition

$$
\omega(\delta) \leqq L\left[\delta \int_{a}^{a / 8} \Omega(x) d x+\int_{1 / 8}^{\infty} \frac{\Omega(x)}{x} d x\right], \quad 0<\delta \leqq 1 / a,
$$

where $a>1$ is a constant independent of $\delta$.

For the case $k=0$, Theorem 3.1 is formulated somewhat differently in [1], but the method of proof employed there can be used to establish the theorem as it is stated above. Inequalities (5.4) and (5.6) in [1, Theorem 5.3] hold as before. The restriction that $q_{n}(\zeta)=v(\zeta)$ of $[1$, Theorem 5.3] is not necessary for the case $k=0$. Under the hypothesis of the present Theorem 3.1, an inequality corresponding to (5.7) of [1, Theorem 5.3], where $p_{n}(z)$ is replaced by $\pi_{n}(z)$, is immediately established, and the theorem follows as before.

It is a consequence of the proof of Theorem 3.1 given in [1] that the only restriction on the constant $a$ is that $\Omega(x)$ be nonincreasing for $x>a$. The method used to prove Theorem 3.1 is a modification of one employed by de la Vallee Poussin to establish results on trigonometric approximation.

We prove now

ThEOREM 3.2. Let $f(z)$ be defined in $\bar{C}$, where $C$ is a curve of Type $\mathrm{D}$; let rational functions $r_{n}(z)$ all of whose poles lie on or exterior to $C_{A}$, $A>1$, exist such that

$$
\left|f(z)-r_{n}(z)\right| \leqq \Omega(n) / n^{n}, \quad z \text { in } \bar{C} .
$$

Then the conclusion of Theorem 3.1 is valid.

Theorem 3.2 has been established by Sewell [2, Theorem 4.2] for the special case that $C$ is an analytic Jordan curve and $\Omega(n)=L n^{-\alpha}$, $0<\alpha \leqq 1$.

Choose $R_{1}$ and $R_{2}$ such that $1<R_{1}<R_{2}<A$. Let $P_{m, n}(z)$ be a polynomial in $z$ of degree $m$ which interpolates to $r_{n}(z)$ in $m+1$ equally- 
distributed points $z_{j}$ on $C_{R_{1}}$. (The " $m+1$ equally-distributed points on $C_{R_{1}}{ }^{n}$ are the images of the $(m+1)$ st roots of unity under the conformal map of $|w|>1$ onto the exterior of $C_{R_{1}}$ by the function $w=\phi(z) / R_{1}$.) The Lagrange-Hermite interpolation formula yields

$$
r_{n}(z)-P_{m, n}(z)=\frac{1}{2 \pi i} \int_{C_{B_{2}}} \frac{\omega_{m}(z) r_{n}(t)}{\omega_{m}(t)(t-z)} d t, \quad z \text { on } C_{R_{1}},
$$

where $\omega_{m}(z)=\left(z-z_{1}\right) \cdots\left(z-z_{m+1}\right)$. For $z$ on $C_{R_{1}}$ and $t$ on $C_{R_{2}}$ $=\left(C_{R_{1}}\right)_{R_{2} / R_{1}}$ we have $\left[4\right.$, Theorem 4.7] that $\left|\omega_{m}(z) / \omega_{m}(t)\right|$ $\leqq M\left(R_{1} / R_{2}\right)^{m}$. It is a consequence of the uniform boundedness of $\left|r_{n}(z)\right|$ and a known result $[3$, p. 250] that

$$
\left|r_{n}(z)\right| \leqq M_{1}\left[\left(A R_{2}-1\right) /\left(A-R_{2}\right)\right]^{n}
$$

for $z$ on $C_{R_{2}}$. Hence

$$
\left|r_{n}(z)-P_{m, n}(z)\right| \leqq M_{2}\left(\frac{R_{1}}{R_{2}}\right)^{m}\left(\frac{A R_{2}-1}{A-R_{2}}\right)^{n}, \quad z \text { on } \bar{C} .
$$

Let $q$ be an integer such that $\left[\left(A R_{2}-1\right) /\left(A-R_{2}\right)\right]\left(R_{1} / R_{2}\right)^{q}=r<1$. It then follows from (3.3) and (3.4) that for $j=0,1, \cdots, q-1$; $n=1,2, \cdots$,

$$
\left|f(z)-P_{q n+j, n}(z)\right| \leqq \Omega(n) / n^{k}+M_{2} r^{n}, \quad z \text { on } \bar{C} .
$$

Since $r^{n}<M_{3} / n^{k+2}$ and $\Omega(n) \leqq \Omega((q n+j) / 2 q)$ for $n$ sufficiently large, we have

$$
\begin{array}{r}
\left|f(z)-P_{q n+j, n}(z)\right| \leqq M_{4}\left[\Omega((q n+j) / 2 q)+1 /(q n+j)^{2}\right] /(q n+j)^{n} \\
z \text { on } \bar{C} .
\end{array}
$$

If we define the polynomials $\pi_{n}(z)$ of Theorem 3.1 as $\pi_{n}(z) \equiv 0$ for $n=1, \cdots, q-1 ; \pi_{q h+j}(z) \equiv P_{q h+j, h}(z)$ for $j=0,1, \cdots, q-1 ; h$ $=1,2, \cdots$, we obtain (3.1) where the $\Omega(x)$ of (3.1) is in terms of the present $\Omega(x), M_{5}\left[\Omega(x / 2 q)+x^{-2}\right]$. Hence it follows from Theorem 3.1 that $f(z)$ is analytic interior to $C$, continuous on $\bar{C}$, and $f^{(k)}(z)$ exists and is continuous on $\bar{C}$ with modulus of continuity $\omega(\delta)$ which satisfies the condition

$$
\omega(\delta) \leqq L\left\{\delta \int_{a}^{a / \delta}\left[\Omega(x / 2 q)+x^{-2}\right] d x+\int_{1 / \delta}^{\infty}\left[\Omega(x / 2 q) / x+x^{-2}\right] d x\right\}
$$

for $0<\delta \leqq 1 / a$, where $a$ is such that $a>2 q$ and $\Omega(x)$ is increasing for $x>a / 2 q$. On suitable change of variable and simplification we obtain an inequality of form (3.2). 


\section{BIBLIOGRAPHY}

1. H. M. Elliott, On approximation to functions satisfying a generalized continuity condition, Trans. Amer. Math. Soc. vol. 71 (1951) pp. 1-23.

2. W. E. Sewell, Continuity and degree of approximation by rational functions, Revista de Ciencias vol. 41 (1939) pp. 435-451.

3. J. L. Walsh, Interpolation and approximation by rational functions in the complex domain, Amer. Math. Soc. Colloquium Publications, vol. 20, New York, 1935.

4. J. L. Walsh and W. E. Sewell, Sufficient conditions for various degrees of approximation by polynomials, Duke Math. J. vol. 6 (1940) pp. 658-705.

WASHINGTON UNIVERSITY

\section{A CONVEX METRIC WITH UNIQUE SEGMENTS}

R. H. BING

1. Introduction. If $D(x, y)$ is a convex metric for a continuous curve $M$ (compact, locally connected, metric continuum), then for each pair of points $p, q$ of $M$ there is an arc $p q$ in $M$ from $p$ to $q$ such that $p q$ is isometric to a straight line interval. We shall call it a segment.

Each continuous curve has a convex metric $[1 ; 3 ; 5 ; 6 ; 2]$. However, if $D(x, y)$ is a convex metric for $M$, there may be two segments from $p$ to $q$. If $M=S_{2}$ is the surface of a sphere, $D(x, y)$ is the size of the central angle subtended by $x$ and $y$, and $p, q$ are diametrically opposite points, then there are many segments from $p$ to $q$. In fact, we show in $\$ 4$ that if $D(x, y)$ is any convex metric whatsoever for $S_{2}$, each point of $S_{2}$ belongs to a pair of points which are not joined by a unique segment.

There is a dense subset $W$ of $S_{2}$ such that no two points of $W$ are diametrically opposite. If $D(x, y)$ is the previously mentioned convex metric for $S_{2}$, then each pair of points of $W$ are joined by one and only one segment. We shall show that for any continuous curve there is such a dense subset and such a convex metric.

Theorem. Each continuous curve $M$ has a dense subset $W$ and a convex metric $D(x, y)$ such that each pair of points of $W$ belongs to a unique segment.

We shall prove this result in much the same manner that it was shown that any continuous curve can be convexified. The metric is

Presented to the Society September 7, 1951; received by the editors March 21, 1952. 